\title{
ON THE CALCULATION OF STOKES MULTIPLIERS FOR LINEAR DIFFERENTIAL EQUATIONS OF THE SECOND ORDER
}

\author{
A. B. Olde Daalhuis and F. W. J. Olver
}

\begin{abstract}
Two new methods are described for the computation of connection coefficients for the asymptotic solutions of linear second-order differential equations having an irregular singularity of arbitrary rank. The first method is based on new asymptotic expansions of the higher coefficients in the asymptotic solutions. The second method is based on direct numerical solution of the differential equation. Numerical examples are included.
\end{abstract}

\section{Introduction}

This paper is concerned with solutions of the homogeneous linear differential equation

$$
\frac{d^{2} w}{d z^{2}}+f(z) \frac{d w}{d z}+g(z) w=0
$$

in the neighborhood of an irregular singularity of positive integer rank $r$. Without loss of generality we may suppose that this singularity is located at $z=\infty$. Then $f(z)$ and $g(z)$ can be expanded in power series of the form

$$
f(z)=z^{r-1} \sum_{s=0}^{\infty} \frac{f_{s}}{z^{s}}, \quad g(z)=z^{2 r-2} \sum_{s=0}^{\infty} \frac{g_{s}}{z^{s}},
$$

that converge on an open annulus $|z|>a$. Moreover, at least one of the coefficients $f_{0}, g_{0}$, and $g_{1}$ is nonzero (otherwise the singularity would be of lower rank).

The nature and the existence of the asymptotic solutions of systerns of homogeneous linear differential equations in the neighborhoods of irregular singularities has been the subject of many investigations; see, for example, $[16,17]$. In the present case, the construction of the asymptotic solutions proceeds as follows [15]. ${ }^{1}$

Let

$$
\phi(z)=\frac{1}{4} f^{2}(z)+\frac{1}{2} f^{\prime}(z)-g(z)
$$

so that

$$
\phi(z)=z^{2 r-2} \sum_{s=0}^{\infty} \frac{h_{s}}{z^{s}}, \quad|z|>a
$$

where

$$
h_{s}=\frac{1}{4}\left(f_{0} f_{s}+f_{1} f_{s-1}+\cdots+f_{s} f_{0}\right)+\frac{1}{2}(2 r-1-s) f_{s-r}-g_{s},
$$

Received October 3, 1994, revised May 11, 1995.

1991 Mathematics Subject Classification. Primary: 34E05, Secondary: 65D30.

Key words and phrases: asymptotic expansions, connection formulas, irregular singularity, numerical integration, remainder term, singulant, Taylor-series method.

The research of the second author was supported by the National Science Foundation under Grant DMS 92-08690.

${ }^{1}$ The present notation differs slightly from that of [15]. 
with $f_{s-r}$ interpreted as being zero when $s<r$. In particular, we have $h_{0}=\frac{1}{4} f_{0}^{2}-g_{0}$. If $h_{0}$ is nonzero, then we can expand the square root of $\phi(z)$ in the form

$$
\{\phi(z)\}^{\frac{1}{2}}=z^{r-1} \sum_{s=0}^{\infty} \frac{\phi_{s}}{z^{s}}
$$

where

$$
\phi_{0}= \pm h_{0}^{\frac{1}{2}}= \pm\left(\frac{1}{4} f_{0}^{2}-g_{0}\right)^{\frac{1}{2}}
$$

and higher coefficients can be computed by means of the recurrence relation

$$
s h_{0} \phi_{s}=\sum_{j=1}^{s}\left(\frac{3}{2} j-s\right) h_{j} \phi_{s-j}, \quad s \geq 1
$$

compare [13], Chapter 1, Exercise 8.4.

With the foregoing definitions, formal series solutions of (1.1) are given by

$$
e^{\xi_{1}(z)} z^{\mu_{1}} \sum_{s=0}^{\infty} \frac{a_{s, 1}}{z^{s}}, \quad e^{\xi_{2}(z)} z^{\mu_{2}} \sum_{s=0}^{\infty} \frac{a_{s, 2}}{z^{s}}
$$

where

$$
\begin{array}{ll}
\xi_{1}(z)=-z^{r} \sum_{s=0}^{r-1} \frac{\phi_{s}+\frac{1}{2} f_{s}}{(r-s) z^{s}}, & \xi_{2}(z)=z^{r} \sum_{s=0}^{r-1} \frac{\phi_{s}-\frac{1}{2} f_{s}}{(r-s) z^{s}} \\
\mu_{1}=-\phi_{r}-\frac{1}{2} f_{r}-\frac{1}{2} r+\frac{1}{2}, & \mu_{2}=\phi_{r}-\frac{1}{2} f_{r}-\frac{1}{2} r+\frac{1}{2}
\end{array}
$$

and the coefficients $a_{s, j}, j=1,2$, are given by $a_{0, j}=1$ and

$$
2 s p_{0, j} a_{s, j}=\sum_{k=1}^{s}\left\{q_{k-1, j}-2(s-k) p_{k, j}\right\} a_{s-k, j}+(s-r)(s-r+1) a_{s-r, j}
$$

the last term being absent when $s \leq r$. Here $p_{k, j}$ and $q_{k, j}$ are the coefficients in the expansions

$$
p_{j}(z)=z^{r-1} \sum_{k=0}^{\infty} \frac{p_{k, j}}{z^{k}}, \quad q_{j}(z)=z^{r-3} \sum_{k=0}^{\infty} \frac{q_{k, j}}{z^{k}}, \quad|z|>a,
$$

of the functions

$$
\begin{aligned}
& p_{j}(z)=\xi_{j}^{\prime}(z)+\frac{\mu_{j}}{z}+\frac{1}{2} f(z), \\
& \begin{aligned}
q_{j}(z)=\xi_{j}^{\prime \prime}(z)+{\xi_{j}^{\prime}}^{2}(z)+ & \frac{2 \mu_{j}}{z} \xi_{j}^{\prime}(z)+\frac{\mu_{j}\left(\mu_{j}-1\right)}{z^{2}} \\
& +f(z)\left\{\xi_{j}^{\prime}(z)+\frac{\mu_{j}}{z}\right\}+g(z) .
\end{aligned}
\end{aligned}
$$

In the exceptional case $\frac{1}{4} f_{0}^{2}=g_{0}$ (which includes, of course, the commonly occurring case of $f_{0}=g_{0}=0$ ), the dominant terms (for large $\left.|z|\right)$ in the polynomials $\xi_{1}(z)$ and $\xi_{2}(z)$ have the same coefficients. If we make the transformation [15]

$$
W=\exp \left\{\frac{1}{2} \int\left(\sum_{s=0}^{r-1} f_{s} z^{r-s-1}\right) d z\right\} w, \quad t=z^{\frac{1}{2}}
$$

then it is readily verified that at $t=\infty$, the transformed differential equation has either a regular singularity or an irregular singularity of rank not exceeding $2 r-1$ with unequal dominant terms in the corresponding polynomials $\xi_{1}(z)$ and $\xi_{2}(z)$. We 
therefore may assume without loss of generality that $\frac{1}{4} f_{0}^{2} \neq g_{0}$ and, hence, that $\phi_{0} \neq 0$ in all subsequent analysis.

The ratio $(s-r)(s-r+1) /\left(2 s p_{0, j}\right)$ of $a_{s, j}$ to $a_{s-r, j}$ in the recurrence relation (1.12) indicates that, in general, the series (1.9) diverge. ${ }^{2}$ In order to describe solutions that have these series as asymptotic expansions, we subdivide the plane into sectors $S_{k}$, $k=0, \pm 1, \pm 2, \ldots$, defined as follows. Let $\sigma=\operatorname{ph} \phi_{0}$; then

$$
S_{k}=\left\{z: \frac{\left(k-\frac{1}{2}\right) \pi-\sigma}{r} \leq \operatorname{ph} z \leq \frac{\left(k+\frac{1}{2}\right) \pi-\sigma}{r}\right\}
$$

see Figure 1.1.

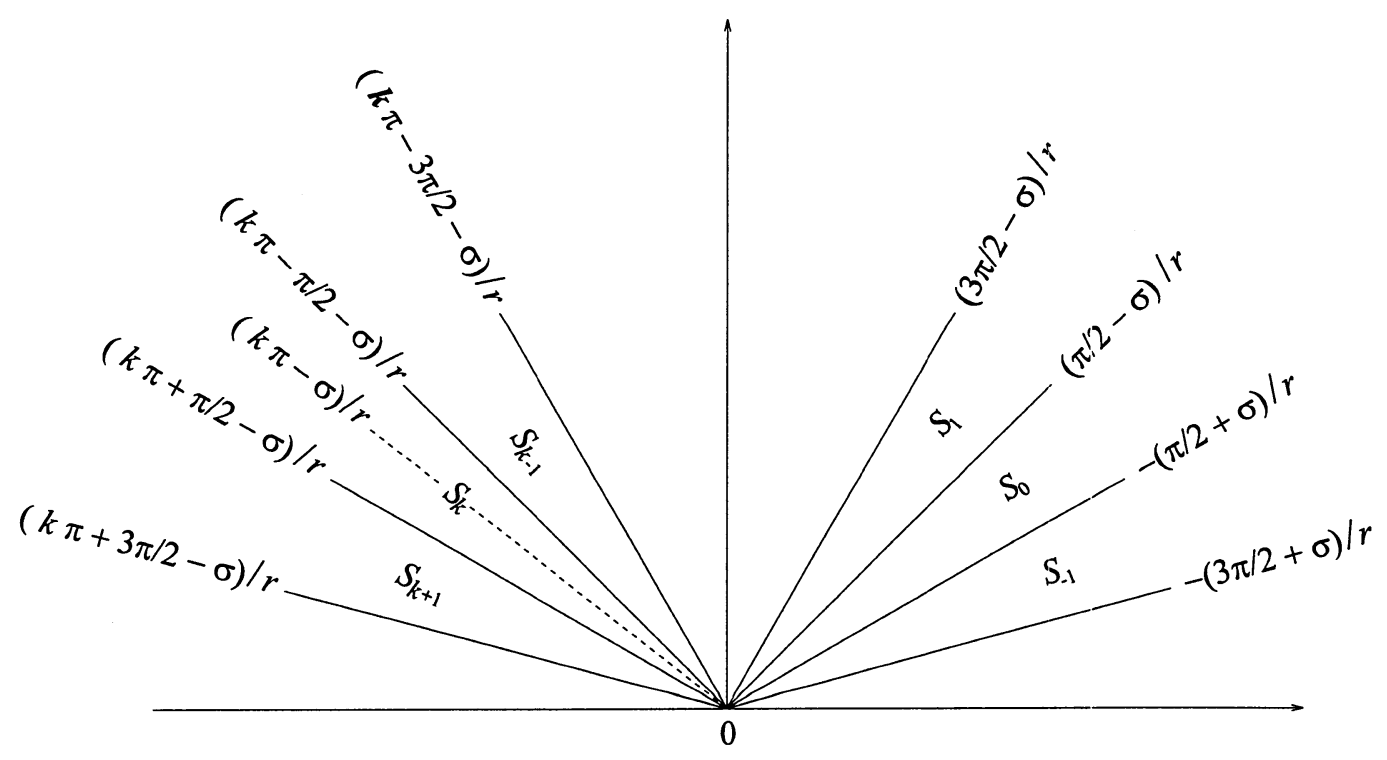

Figure 1.1. $z$-plane. Sectors $S_{k}$. Values of $\mathrm{ph} z$ are given at ends of rays.

The significant feature of these sectors is that for sufficiently large $|z|$, the real part of $\xi_{2}(z)-\xi_{1}(z)$ is positive or negative at interior points of $S_{k}$ according as $k$ is even or odd. This follows from the relation

$$
\xi_{2}(z)-\xi_{1}(z) \sim 2 \phi_{0} z^{r} / r, \quad z \rightarrow \infty
$$

obtained from (1.10).

Now define $\hat{S}_{k}$ to be any closed sector that is properly interior to $S_{k-1} \cup S_{k} \cup S_{k+1}$. For example, $\hat{S}_{0}$ could be taken to be the sector

$$
-\left(\frac{3}{2} \pi+\sigma-\delta\right) / r \leq \operatorname{ph} z \leq\left(\frac{3}{2} \pi-\sigma-\delta\right) / r,
$$

where $\delta$ is an arbitrary small positive constant.

Theorem 1.1. (i) For each even integer $k$, there exists a unique solution $w_{k}(z)$ of (1.1) such that

$$
w_{k}(z) \sim e^{\xi_{1}(z)} z^{\mu_{1}} \sum_{s=0}^{\infty} \frac{a_{s, 1}}{z^{s}}, \quad z \rightarrow \infty \text { in } \hat{S}_{k} .
$$

\footnotetext{
${ }^{2}$ See also Theorem 4.1 below.
} 
(ii) For each odd integer $k$, there exists a unique solution $w_{k}(z)$ of (1.1) such that

$$
w_{k}(z) \sim e^{\xi_{2}(z)} z^{\mu_{2}} \sum_{s=0}^{\infty} \frac{a_{s, 2}}{z^{s}}, \quad z \rightarrow \infty \text { in } \hat{S}_{k} .
$$

Any branches may be chosen for $\sigma \equiv \mathrm{ph} \phi_{0}$ in (1.15) and $z^{\mu_{1}}, z^{\mu_{2}}$ in (1.16), provided that they are used consistently.

Theorem 1.1 can be derived from the theorem of [15] by suitable choice of the path $\mathcal{P}_{k}$ in this theorem. Thus, in the present notation, if we take $\hat{S}_{k}$ to be the sector

$$
\hat{S}_{k}=\left\{z: \frac{\left(k-\frac{3}{2}\right) \pi-\sigma+\delta}{r} \leq \operatorname{ph} z \leq \frac{\left(k+\frac{3}{2}\right) \pi-\sigma-\delta}{r}\right\}
$$

and make the transformation $\zeta=\phi_{0} z^{r}$, then for all sufficiently large $z$ in $\hat{S}_{k}$, the path $\mathcal{P}_{k}$ can be taken as the map of a path $\mathcal{Q}_{k}$ in the $\zeta$-plane comprising an infinite segment of the real axis and segments of one or two straight lines at angles $\pm \frac{1}{2} \delta$ to the imaginary axis. Figures 1.2, 1.3, for example, depict $\mathcal{Q}_{k}$ when $k$ is even and $(k \pi-\sigma) / r \leq \operatorname{ph} z \leq\left(k \pi+\frac{1}{2} \pi-\sigma\right) / r,\left(k \pi+\frac{1}{2} \pi-\sigma\right) / r \leq \operatorname{ph} z \leq\left(k \pi+\frac{3}{2} \pi-\sigma-\delta\right) / r$, respectively. See also Lemma 13.1 of [13], Chapter 6 .

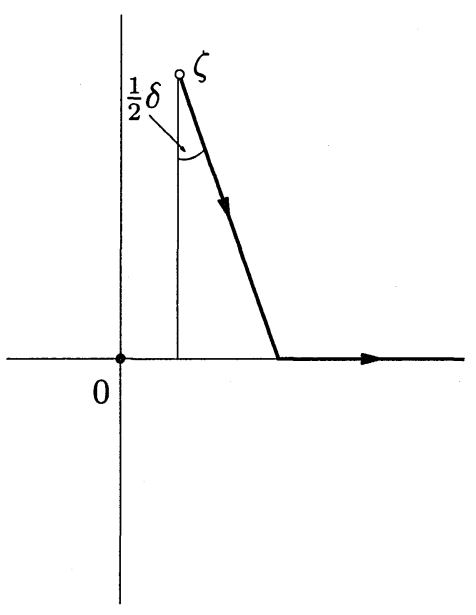

Figure 1.2. $\zeta$-plane. Path $\mathcal{Q}_{k}$ when $k \pi \leq \operatorname{ph} \zeta \leq\left(k+\frac{1}{2}\right) \pi, k$ even.

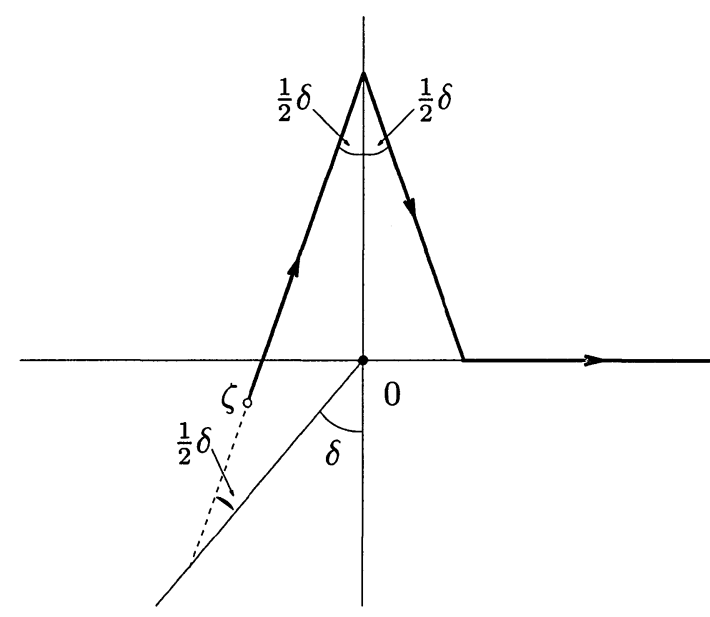

Figure 1.3. $\zeta$-plane. Path $\mathcal{Q}_{k}$ when $\left(k+\frac{1}{2}\right) \pi \leq \operatorname{ph} \zeta \leq\left(k+\frac{3}{2}\right) \pi-\delta, k$ even.

\section{The connection formula problem}

Although, for each value of $k$, Theorem 1.1 provides information on the solution $w_{k}(z)$ only in the sector $\hat{S}_{k}$, this solution can be continued analytically to all values of $\mathrm{ph} z$. In general, $w_{k}(z)$ has a branch-point at infinity.

Among any three solutions a linear relation holds for all values of $z$. In particular,

$$
w_{k+1}(z)=C_{k} w_{k}(z)+B_{k} w_{k-1}(z),
$$

where $B_{k}$ and $C_{k}$ are constants. The value of $B_{k}$ is easily found by letting $z \rightarrow \infty$ on an interior ray in $S_{k}$. On this ray, $w_{k}(z)$ is recessive and the other two solutions are 
dominant with exactly the same asymptotic form. Hence $B_{k}=1$, and (2.1) reduces to

$$
w_{k+1}(z)=C_{k} w_{k}(z)+w_{k-1}(z) .
$$

The coefficient $C_{k}$ is more difficult to calculate in general. For later convenience, we sometimes set

$$
C_{k}=e^{(-)^{k} k \omega \pi i / r} A_{k}
$$

where

$$
\omega=\mu_{2}-\mu_{1}
$$

then

$$
w_{k+1}(z)=e^{(-)^{k} k \omega \pi i / r} A_{k} w_{k}(z)+w_{k-1}(z) .
$$

Either set of constants $\left\{C_{k}\right\}$ or $\left\{A_{k}\right\}, k=0, \pm 1, \ldots$, may be called the Stokes multipliers (at infinity) for the differential equation (1.1).

In passing, we note that if we know the $C_{k}$ (or $A_{k}$ ) for all values of $k$, then it is possible to determine the asymptotic behavior of any solution $w_{j}(z)$, say, in any sector $S_{k}$, say. Suppose, for example, $k>j$. It is readily verified that

$$
w_{j}(z)=\Lambda_{k} w_{k-1}(z)+\Lambda_{k-1} w_{k}(z),
$$

where the constants $\Lambda_{k-1}$ and $\Lambda_{k}$ are found by application of the recurrence relation

$$
\Lambda_{n+1}=-C_{n} \Lambda_{n}+\Lambda_{n-1}, \quad n=j+2, j+3, \ldots, k-1,
$$

beginning with $\Lambda_{j+1}=1$ and $\Lambda_{j+2}=-C_{j+1}$. Once $\Lambda_{k}$ and $\Lambda_{k-1}$ have been found, the (compound) asymptotic expansion of $w_{j}(z)$ in the sector $\hat{S}_{k-1} \cap \hat{S}_{k}$ is obtained from (2.6) by substituting the known expansions (1.16) of $w_{k-1}(z)$ and $w_{k}(z)$ in this sector. Obviously from Figure 1.1, we can always arrange that $\hat{S}_{k-1} \cap \hat{S}_{k}$ includes the sector

$$
\left(k \pi-\frac{1}{2} \pi-\sigma\right) / r \leq \operatorname{ph} z \leq(k \pi-\sigma) / r,
$$

that is, the right half of $S_{k}$ when viewed from the origin. To cover the left half of $S_{k}$, we continue the recurrence (2.7) one step further, to $n=k$, and then apply

$$
w_{j}(z)=\Lambda_{k+1} w_{k}(z)+\Lambda_{k} w_{k+1}(z) \text {. }
$$

The purpose of the present paper is to describe two methods for computing the coefficients $C_{k}$ and $A_{k}$ in the connection formulas (2.2) and (2.5), one asymptotic, the other numerical.

The asymptotic method is developed in $\S \S 3,4,5$ and is based on expansions of the coefficients $a_{s, 1}$ and $a_{s, 2}$ for large $s$. Methods of this kind are already known; see, for example, [6-11]. Other analytical methods also exist $[5,18]$. The novelty of our approach is that we employ inverse factorial series in place of the more usual inverse power series for the asymptotic expansions of $a_{s, 1}$ and $a_{s, 2}$. The advantage of the inverse factorial expansions is that the coefficients are available explicitly. In fact, they are simple multiples of the earlier coefficients $a_{0,1}, a_{1,1}, a_{2,1}, \ldots, a_{0,2}, a_{1,2}, a_{2,2}, \ldots$ Therefore, it is possible to evaluate the Stokes multipliers to high accuracy with relatively little analytical and computational effort. In $\S 6$, we supply two numerical examples to illustrate this feature.

The second method for evaluating the Stokes multipliers is based on direct numerical integration of the differential equation along suitably chosen paths in the complex plane. It is outlined in $\S 7$ and applied to the numerical examples previously treated in $\S 6$. 


\section{Asymptotic method for the Stokes multipliers: singularities of unit rank}

In this section, we assume that $r=1$. The expressions (1.10) for $\xi_{1}(z)$ and $\xi_{2}(z)$ reduce to

$$
\xi_{1}(z)=\lambda_{1} z, \quad \xi_{2}(z)=\lambda_{2} z
$$

where $\lambda_{1}, \lambda_{2}$ are the zeros of the quadratic polynomial $\lambda^{2}+f_{0} \lambda+g_{0}$. Accordingly,

$$
\lambda_{2}-\lambda_{1}= \pm\left(f_{0}^{2}-4 g_{0}\right)^{\frac{1}{2}}
$$

and is nonzero by hypothesis $(\S 1)$. The exponents $\mu_{1}$ and $\mu_{2}$ are given by

$$
\mu_{1}=\frac{f_{1} \lambda_{1}+g_{1}}{\lambda_{2}-\lambda_{1}}, \quad \mu_{2}=\frac{f_{1} \lambda_{2}+g_{1}}{\lambda_{1}-\lambda_{2}},
$$

and the recurrence relation (1.12), with $j=1,2$, respectively, reduces to

$$
\begin{aligned}
\left(\lambda_{1}-\lambda_{2}\right) s a_{s, 1}= & \left(s-\mu_{1}\right)\left(s-1-\mu_{1}\right) a_{s-1,1} \\
& \quad+\sum_{j=1}^{s}\left\{\lambda_{1} f_{j+1}+g_{j+1}-\left(s-j-\mu_{1}\right) f_{j}\right\} a_{s-j, 1}, \\
\left(\lambda_{2}-\lambda_{1}\right) s a_{s, 2}= & \left(s-\mu_{2}\right)\left(s-1-\mu_{2}\right) a_{s-1,2} \\
& \quad+\sum_{j=1}^{s}\left\{\lambda_{2} f_{j+1}+g_{j+1}-\left(s-j-\mu_{2}\right) f_{j}\right\} a_{s-j, 2},
\end{aligned}
$$

again with the starting values $a_{0,1}=a_{0,2}=1$. We also have $\phi_{0}=\frac{1}{2}\left(\lambda_{2}-\lambda_{1}\right)$, $\sigma=\operatorname{ph}\left(\lambda_{2}-\lambda_{1}\right)$. The sectors $S_{k}, k=0, \pm 1, \ldots$, are defined by

$$
S_{k}=\left\{z:\left(k-\frac{1}{2}\right) \pi-\sigma \leq \mathrm{ph} z \leq\left(k+\frac{1}{2}\right) \pi-\sigma\right\} .
$$

Next, we observe that $w_{k}\left(e^{-2 j \pi i} z\right)$ is also a solution of the differential equation for any integer $j$, and from Theorem 1.1 it follows that

$$
\begin{aligned}
& w_{k+2 j}(z)=e^{2 j \mu_{1} \pi i} w_{k}\left(e^{-2 j \pi i} z\right), \quad k \text { even, } \\
& w_{k+2 j}(z)=e^{2 j \mu_{2} \pi i} w_{k}\left(e^{-2 j \pi i} z\right), \quad k \text { odd. }
\end{aligned}
$$

On combining these results with (2.5), with $r=1$, and (2.4), we deduce that

$$
A_{k}=A_{0}, \quad k \text { even; } \quad A_{k}=A_{1}, \quad k \text { odd. }
$$

Now suppose that the differential equation is normalized in such a way that

$$
\lambda_{2}-\lambda_{1}=1 \text {. }
$$

This always can be arranged, if necessary, by replacing $z$ with $z /\left(\lambda_{2}-\lambda_{1}\right)$. Then $\phi_{0}=\frac{1}{2}, \sigma=0$, and the sectors $S_{k}$ become

$$
S_{k}=\left\{z:\left(k-\frac{1}{2}\right) \pi \leq \operatorname{ph} z \leq\left(k+\frac{1}{2}\right) \pi\right\} .
$$

Furthermore, the new values of $C_{k}$ and $A_{k}$ are $\left(\lambda_{2}-\lambda_{1}\right)^{(-)^{k} \omega}$ times their old values. ${ }^{3}$

In [12], we proved that in the present circumstances the following theorem holds. ${ }^{4}$

\footnotetext{
${ }^{3}$ In forming $\left(\lambda_{2}-\lambda_{1}\right)^{(-)^{k} \omega}$, the branch of $\mathrm{ph}\left(\lambda_{2}-\lambda_{1}\right)$ has to be $\sigma$.

${ }^{4}$ The notations $w_{1}(z), w_{2}(z), C_{1}$, and $C_{2}$ in [12] now are denoted by $w_{0}(z), w_{1}(z),-C_{1}$, and $C_{0}$, respectively.
} 
Theorem 3.1. Let $m$ be an arbitrary fixed nonnegative integer. Then as $s \rightarrow \infty$,

$$
\begin{aligned}
& a_{s, 1}=(-)^{s-1} \frac{A_{1}}{2 \pi i} \sum_{j=0}^{m-1}(-)^{j} a_{j, 2} \Gamma(s+\omega-j)+\mathcal{O}\{\Gamma(s+\omega-m)\}, \\
& a_{s, 2}=-\frac{A_{0}}{2 \pi i} \sum_{j=0}^{m-1} a_{j, 1} \Gamma(s-\omega-j)+\mathcal{O}\{\Gamma(s-\omega-m)\}
\end{aligned}
$$

where $\omega$ is defined by (2.4).

Equations (3.7) can be regarded in two ways. If $A_{0}$ and $A_{1}$ are known, then (3.7) provide an easy way of computing $a_{s, 1}$ and $a_{s, 2}$ for large $s$.

Alternatively, if we regard equations (3.4) as a way of generating $a_{s, 1}$ and $a_{s, 2}$ recursively for arbitrary large $s$, then $A_{0}$ and $A_{1}$ can be estimated via

$$
A_{0}=-2 \pi i \lim _{s \rightarrow \infty} \frac{a_{s, 2}}{\Gamma(s-\omega)}, \quad A_{1}=2 \pi i \lim _{s \rightarrow \infty} \frac{(-)^{s-1} a_{s, 1}}{\Gamma(s+\omega)}
$$

or more accurately from

$$
\begin{gathered}
A_{0}=-2 \pi i a_{s, 2}\left\{\sum_{j=0}^{m-1} a_{j, 1} \Gamma(s-\omega-j)\right\}^{-1}+\mathcal{O}\left(s^{-m}\right), \\
A_{1}=(-)^{s-1} 2 \pi i a_{s, 1}\left\{\sum_{j=0}^{m-1}(-)^{j} a_{j, 2} \Gamma(s+\omega-j)\right\}^{-1}+\mathcal{O}\left(s^{-m}\right) .
\end{gathered}
$$

It is the second point of view that we adopt in this paper. By choosing values of $s$ and $m$ that are large (but not unduly large), it is possible to compute $A_{0}$ and $A_{1}$ from (3.9) to very high accuracy.

\section{Asymptotic method for the Stokes multipliers: singularities of arbitrary rank}

We now proceed to the generalization of Theorem 3.1 to the case of arbitrary rank $r$.

Corresponding to (3.5), we have

$$
\begin{array}{ll}
w_{k+2 j r}(z)=e^{2 j \mu_{1} \pi i} w_{k}\left(e^{-2 j \pi i} z\right), & k \text { even, } \\
w_{k+2 j r}(z)=e^{2 j \mu_{2} \pi i} w_{k}\left(e^{-2 j \pi i} z\right), & k \text { odd } .
\end{array}
$$

From these results and (2.4), (2.5), it follows that

$$
A_{k+2 j r}=A_{k},
$$

for all integers $k$ and $j$. Accordingly, it suffices to determine a set of $2 r$ consecutive Stokes multipliers.

With $\xi_{1}(z)$ and $\xi_{2}(z)$ defined as in $\S 1$, we follow the terminology of Dingle [3], and call $\xi_{2}(z)-\xi_{1}(z)$ the singulant (with respect to $z=\infty$ ) of the differential equation (1.1). The first step is to renormalize the differential equation in such a way that the singulant is simply $z^{r}$; compare (3.1) and (3.6) in the case $r=1$. This can be achieved in various ways. The simplest, in principle, is to take a new independent variable $x$ defined by

$$
x^{r}=\xi_{2}(z)-\xi_{1}(z)=2 z^{r} \sum_{s=0}^{r-1} \frac{\phi_{s}}{(r-s) z^{s}}
$$


compare (1.10). For large $x$, we can expand $z$ in a convergent power series of the form

$$
z=x \sum_{s=0}^{\infty} \frac{c_{s}}{x^{s}}
$$

where, for example,

$$
\begin{gathered}
c_{0}=\left(\frac{r}{2 \phi_{0}}\right)^{1 / r}, \quad c_{1}=-\frac{\phi_{1}}{(r-1) \phi_{0}}, \\
c_{2}=\frac{\phi_{1}^{2}}{2(r-1) \phi_{0}^{2} c_{0}}-\frac{\phi_{2}}{(r-2) \phi_{0} c_{0}},
\end{gathered}
$$

and higher coefficients can be found by means of standard procedures for reversion of power series. The problem with this approach is that reversion is cumbersome; even with the aid of available software packages, the evaluation of $c_{s}$ is slow when $s$ is large. Following [10], Section III.3, we circumvent this difficulty by truncating the expansion (4.4) after $r$ terms. Thus, instead of $x$, we use the variable $\tilde{z}$ defined implicitly by

$$
z=\tilde{z} \sum_{s=0}^{r-1} \frac{c_{s}}{\tilde{z}^{s}}
$$

with the condition $c_{0} \tilde{z} \sim z$ as $z \rightarrow \infty$. The effect of this modification is to replace (4.3) by

$$
\xi_{2}(z)-\xi_{1}(z)=\tilde{z}^{r}-\frac{r c_{r}}{c_{0}}+\mathcal{O}\left(\frac{1}{\tilde{z}}\right)
$$

the term $\mathcal{O}(1 / \tilde{z})$ here actually being a power series in $1 / \tilde{z}$.

In terms of the variable $\tilde{z}$, the differential equation (1.1) transforms into

$$
\frac{d^{2} w}{d \tilde{z}^{2}}+\tilde{f}(\tilde{z}) \frac{d w}{d \tilde{z}}+\tilde{g}(\tilde{z}) w=0
$$

where

$$
\tilde{f}(\tilde{z})=-\left(\frac{d z}{d \tilde{z}}\right)^{-1} \frac{d^{2} z}{d \tilde{z}^{2}}+\frac{d z}{d \tilde{z}} f(z), \quad \tilde{g}(\tilde{z})=\left(\frac{d z}{d \tilde{z}}\right)^{2} g(z) .
$$

From (1.2) and (4.6), we see that $\tilde{f}(\tilde{z})$ and $\tilde{g}(\tilde{z})$ can be expanded in the form

$$
\tilde{f}(\tilde{z})=\tilde{z}^{r-1} \sum_{s=0}^{\infty} \frac{\tilde{f}_{s}}{\tilde{z}^{s}}, \quad \tilde{g}(\tilde{z})=\tilde{z}^{2 r-2} \sum_{s=0}^{\infty} \frac{\tilde{g}_{s}}{\tilde{z}^{s}},
$$

these series converging for all sufficiently large values of $|\tilde{z}|$. In particular, we have

$$
\tilde{f}_{0}=c_{0}^{r} f_{0}, \quad \tilde{g}_{0}=c_{0}^{2 r} g_{0}, \quad \tilde{g}_{1}=c_{0}^{2 r-1}\left\{g_{1}+(2 r-2) c_{1} g_{0}\right\} .
$$

Since not all of $f_{0}, g_{0}$, and $g_{1}$ vanish, the same is true of $\tilde{f}_{0}, \tilde{g}_{0}$, and $\tilde{g}_{1}$. Furthermore,

$$
\tilde{\phi}_{0} \equiv\left(\frac{1}{4} \tilde{f}_{0}^{2}-\tilde{g}_{0}\right)^{\frac{1}{2}}=c_{0}^{r} \phi_{0}=\frac{1}{2} r .
$$

On applying Theorem 1.1, we see that if $\tilde{S}_{k}$ denotes the sector

$$
\frac{\left(k-\frac{3}{2}\right) \pi+\delta}{r} \leq \operatorname{ph} \tilde{z} \leq \frac{\left(k+\frac{3}{2}\right) \pi-\delta}{r},
$$

then equation (4.8) has solutions $\tilde{w}_{k}(\tilde{z})$ such that, as $\tilde{z} \rightarrow \infty$ in $\tilde{S}_{k}$,

$$
\tilde{w}_{k}(\tilde{z}) \sim e^{\tilde{\xi}_{1}(\tilde{z})} \tilde{z}^{\tilde{\mu}_{1}} \quad \text { or } \quad \tilde{w}_{k}(\tilde{z}) \sim e^{\tilde{\xi}_{2}(\tilde{z})} \tilde{z}^{\tilde{\mu}_{2}}
$$


according as $k$ is even or odd. Furthermore, because $\tilde{w}_{k}(\tilde{z})$ is recessive when $\tilde{z} \rightarrow \infty$ in the central third of $\tilde{S}_{k}$ and $w_{k}(z)$ is recessive when $z \rightarrow \infty$ in the central third of $\hat{S}_{k}$, it follows that

$$
\begin{gathered}
\tilde{\mu}_{1}=\mu_{1}, \quad \tilde{\mu}_{2}=\mu_{2}, \\
\xi_{1}(z)-\tilde{\xi}_{1}(\tilde{z})=b_{1}+\mathcal{O}\left(\tilde{z}^{-1}\right), \quad \xi_{2}(z)-\tilde{\xi}_{2}(\tilde{z})=b_{2}+\mathcal{O}\left(\tilde{z}^{-1}\right), \quad \tilde{z} \rightarrow \infty,
\end{gathered}
$$

where $b_{1}, b_{2}$ are constants and, hence,

$$
w_{k}(z)=e^{b_{1}} c_{0}^{\mu_{1}} \tilde{w}_{k}(\tilde{z}) \quad \text { or } \quad w_{k}(z)=e^{b_{2}} c_{0}^{\mu_{2}} \tilde{w}_{k}(\tilde{z}),
$$

according as $k$ is even or odd. Next, since $\tilde{\xi}_{1}(\tilde{z})$ and $\tilde{\xi}_{2}(\tilde{z})$ are polynornials in $\tilde{z}$ of degree $r$ with no constant term, it follows from (4.7) and (4.12) that $\tilde{\xi}_{2}(\tilde{z})-\tilde{\xi}_{1}(\tilde{z})=\tilde{z}^{r}$, exactly (as required), and also that

$$
b_{1}-b_{2}=\frac{r c_{r}}{c_{0}}
$$

Lastly, let $\left\{\tilde{A}_{k}\right\}$ be the set of Stokes multipliers associated with (4.8) at $\tilde{z}=\infty$, so that

$$
\tilde{w}_{k+1}(\tilde{z})=e^{(-)^{k} k \omega \pi i / r} \tilde{A}_{k} \tilde{w}_{k}(\tilde{z})+\tilde{w}_{k-1}(\tilde{z}),
$$

with $\omega$ again defined by (2.4); compare (2.5) and (4.11). Then from (4.13) and (4.14), we deduce that these multipliers are related to the original multipliers $A_{k}$ by the formula ${ }^{5}$

$$
A_{k}=e^{(-)^{k-1} r c_{r} / c_{0}} c_{0}^{(-)^{k} \omega} \tilde{A}_{k} .
$$

From here until the end of $\S 5$, we shall assume that the preliminary transformation of the form (4.6) has been made, if needed; in consequence, the normalizing condition

$$
\xi_{2}(z)-\xi_{1}(z)=z^{r}
$$

is satisfied.

We observe in passing that when (4.17) holds, the definitions of $\$ 1$ simplify, in that $\phi_{0}=\frac{1}{2} r, \phi_{s}=0 \quad(1 \leq s \leq r-1)$, and $\sigma=0$.

Theorem 4.1. Let $m$ be an arbitrary fixed nonnegative integer. Then with the condition (4.17), the coefficients $a_{s, 1}$ and $a_{s, 2}$ defined in $\S 1$ have the asymptotic expansions as $s \rightarrow \infty$

$$
\begin{aligned}
a_{s, 1}=-\frac{1}{2 r \pi i} \sum_{k=0}^{r-1} A_{2 k+1} \sum_{j=0}^{m-1} a_{j, 2} e^{(2 k+1)(s-j) \pi i / r} & \Gamma\left(\frac{s+\omega-j}{r}\right) \\
& +\mathcal{O}\left\{\Gamma\left(\frac{s+\omega-m}{r}\right)\right\}, \\
a_{s, 2}=-\frac{1}{2 r \pi i} \sum_{k=0}^{r-1} A_{2 k} \sum_{j=0}^{m-1} a_{j, 1} e^{2 k(s-j) \pi i / r} & \Gamma\left(\frac{s-\omega-j}{r}\right) \\
& +\mathcal{O}\left\{\Gamma\left(\frac{s-\omega-m}{r}\right)\right\},
\end{aligned}
$$

where $\omega=\mu_{2}-\mu_{1}$.

\footnotetext{
${ }^{5}$ Again, the branch of $\mathrm{ph} c_{0}$ used in forming $c_{0}^{(-)^{k} \omega}$ in (4.16) is $-\left(\mathrm{ph} \phi_{0}\right) / r$, where $\phi_{0}$ pertains to the original equation (1.1).
} 
This theorem is proved in $\S 5$. In the remainder of the present section, we describe how it can be applied to compute the $A_{k}$.

The $\mathcal{O}$-terms on the right-hand sides of (4.18) contribute relative errors $\mathcal{O}\left(s^{-m / r}\right)$ as $s \rightarrow \infty$. We assume that $s$ and $m$ are chosen sufficiently large to render these contributions negligible compared with the desired precision in the $A_{k}$. On replacing $s$ in $(4.18 \mathrm{a})$ by $r s, r s+1, \ldots, r s+r-1$, in turn, and ignoring the error terms, we arrive at the system of $r$ equations for $A_{1}, A_{3}, \ldots, A_{2 r-1}$

$$
\begin{gathered}
\sum_{k=0}^{r-1} b_{0,2 k+1} A_{2 k+1}=a_{r s, 1} \\
\sum_{k=0}^{r-1} b_{1,2 k+1} A_{2 k+1}=a_{r s+1,1} \\
\vdots \\
\sum_{k=0}^{r-1} b_{r-1,2 k+1} A_{2 k+1}=a_{r s+r-1,1}
\end{gathered}
$$

where

$$
b_{n, 2 k+1}=\frac{(-)^{s-1}}{2 r \pi i} e^{(2 k+1) n \pi i / r} \sum_{j=0}^{m-1} a_{j, 2} e^{-(2 k+1) j \pi i / r} \Gamma\left(s+\frac{n+\omega-j}{r}\right)
$$

In a similar way, we find that

$$
\begin{gathered}
\sum_{k=0}^{r-1} b_{0,2 k} A_{2 k}=a_{r s, 2}, \\
\sum_{k=0}^{r-1} b_{1,2 k} A_{2 k}=a_{r s+1,2}, \\
\vdots \\
\sum_{k=0}^{r-1} b_{r-1,2 k} A_{2 k}=a_{r s+r-1,2}
\end{gathered}
$$

where

$$
b_{n, 2 k}=-\frac{1}{2 r \pi i} e^{2 k n \pi i / r} \sum_{j=0}^{m-1} a_{j, 1} e^{-2 k j \pi i / r} \Gamma\left(s+\frac{n-\omega-j}{r}\right) .
$$

Each system (4.19), (4.21) may be solved by standard numerical procedures for linear algebraic equations. To investigate the conditioning of these systems, we replace each coefficient $b_{n, k}$ by the corresponding dominant term on the right-hand side of (4.20) or (4.22), that is, we take $m=1$. In this way, we find that (4.19) has the 
approximate form

$$
\begin{aligned}
& A_{1}+A_{3}+\cdots+\quad A_{2 r-1} \quad=\quad(-)^{s-1} \frac{2 r \pi i a_{r s, 1}}{\Gamma\left(s+\frac{\omega}{r}\right)}, \\
& e^{\pi i / r} A_{1}+e^{3 \pi i / r} A_{3}+\cdots+e^{(2 r-1) \pi i / r} A_{2 r-1}=(--)^{s-1} \frac{2 r \pi i a_{r s+1,1}}{\Gamma\left(s+\frac{1+\omega}{r}\right)}, \\
& e^{(r-1) \pi i / r} A_{1}+e^{(r-1) 3 \pi i / r} A_{3}+\cdots+e^{(r-1)(2 r-1) \pi i / r} A_{2 r-1}=(-)^{s-1} \frac{2 r \pi i a_{r s+r-1,1}}{\Gamma\left(s+\frac{r-1+\omega}{r}\right)} .
\end{aligned}
$$

This is a Vandermonde system of equations, and its condition number in the infinity norm is easily deduced from the result of [4, Example 6.4] to be $r$. Hence the system (4.19) is well conditioned. In a similar way, we may show that the same is true for (4.21).

\section{Proof of Theorem 4.1}

The proof of Theorem 4.1 is an extension of that of Theorem 2.1 in [12]. With the solutions $w_{k}(z)$ defined as in $\S 1$, we set

$$
v_{k}(z)=e^{-\xi_{1}(z)} z^{-\mu_{1}-1} w_{k}(z) .
$$

Then

$$
v_{k+1}(z)=C_{k} v_{k}(z)+v_{k-1}(z)
$$

compare (2.2).

Lemma 5.1. Let $\rho$ be any constant that exceeds $a$. Then

$$
2 \pi i v_{0}(z)=\sum_{k=0}^{r-1} C_{2 k+1} \int_{\rho e^{(2 k+1) \pi i / r}}^{\infty e^{(2 k+1) \pi i / r}} \frac{v_{2 k+1}(t)}{t-z} d t-\sum_{k=0}^{r-1} \int_{\rho e^{(2 k-1) \pi i / r}}^{\rho e^{(2 k+1) \pi i / r}} \frac{v_{2 k}(t)}{t-z} d t
$$

valid when $z$ lies in the annular sector $|z|>\rho,|\operatorname{ph} z|<\pi / r$.

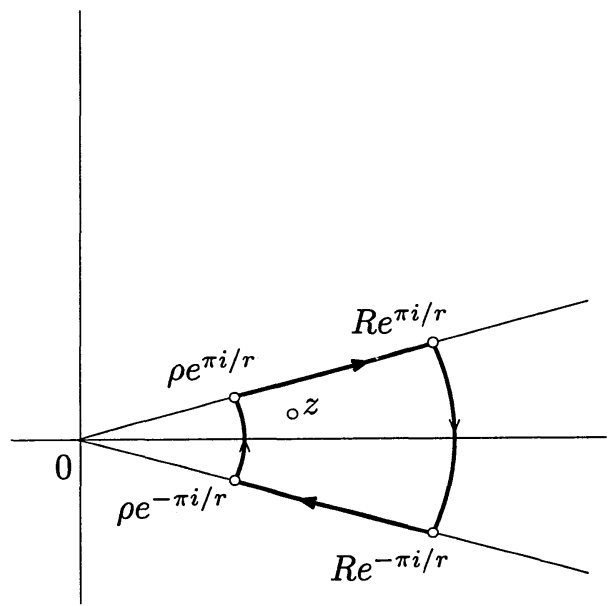

Figure 5.1. $t$-plane. Contour $\mathcal{C}_{0}$.

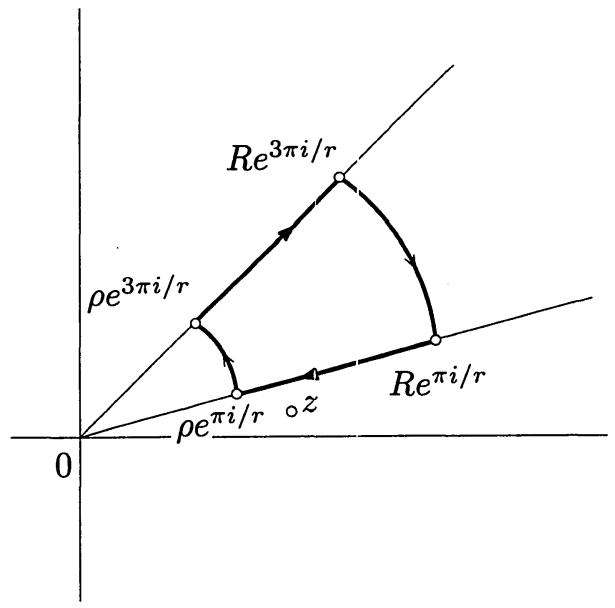

Figure 5.2. $t$-plane. Contour $\mathcal{C}_{1}$. 
Proof. Let $R$ be any constant that exceeds $|z|$. Then, from Cauchy's integral formula, we have

$$
2 \pi i v_{0}(z)=-\int_{\mathcal{C}_{0}} \frac{v_{0}(t)}{t-z} d t
$$

where $\mathcal{C}_{0}$ is the closed contour depicted in Figure 5.1. From (1.16a) and (5.1), with $k=0$, we see that $v_{0}(t)=\mathcal{O}\left(t^{-1}\right)$ uniformly as $t \rightarrow \infty$ in the sector $|\operatorname{ph} t| \leq \pi / r$. Accordingly, on letting $R \rightarrow \infty$, (5.4) becomes

$$
2 \pi i v_{0}(z)=-\left(\int_{\rho e^{\pi i / r}}^{\infty e^{\pi i / r}}-\int_{\rho e^{-\pi i / r}}^{\infty e^{-\pi i / r}}+\int_{\rho e^{-\pi i / r}}^{\rho e^{\pi i / r}}\right) \frac{v_{0}(t)}{t-z} d t
$$

In the first of these integrals, we substitute for $v_{0}(t)$ by means of (5.2) with $k=1$. This yields

$$
\begin{aligned}
2 \pi i v_{0}(z)=C_{1} \int_{\rho e^{\pi i / r}}^{\infty e^{\pi i / r}} \frac{v_{1}(t)}{t-z} d t-\int_{\rho e^{-\pi i / r}}^{\rho e^{\pi i / r}} \frac{v_{0}(t)}{t-z} d t \\
\quad+\int_{\rho e^{-\pi i / r}}^{\infty e^{-\pi i / r}} \frac{v_{0}(t)}{t-z} d t-\int_{\rho e^{\pi i / r}}^{\infty e^{\pi i / r}} \frac{v_{2}(t)}{t-z} d t
\end{aligned}
$$

We now integrate $v_{2}(t) /(t-z)$ around the closed contour $\mathcal{C}_{1}$ depicted in Figure 5.2, and again let $R \rightarrow \infty$. From (1.16a) and (5.1), with $k=2$, we see that $v_{2}(t)=\mathcal{O}\left(t^{-1}\right)$ uniformly as $t \rightarrow \infty$ in the sector $\pi / r \leq \operatorname{ph} t \leq 3 \pi / r$. Hence, we obtain

$$
\int_{\rho e^{\pi i / r}}^{\infty e^{\pi i / r}} \frac{v_{2}(t)}{t-z} d t=\int_{\rho e^{3 \pi i / r}}^{\infty e^{3 \pi i / r}} \frac{v_{2}(t)}{t-z} d t+\int_{\rho e^{\pi i / r}}^{\rho e^{3 \pi i / r}} \frac{v_{2}(t)}{t-z} d t
$$

Then by application of (5.2), with $k=3$, we derive

$$
\int_{\rho e^{\pi i / r}}^{\infty e^{\pi i / r}} \frac{v_{2}(t)}{t-z} d t=-C_{3} \int_{\rho e^{3 \pi i / r}}^{\infty e^{3 \pi i / r}} \frac{v_{3}(t)}{t-z} d t+\int_{\rho e^{3 \pi i / r}}^{\infty e^{3 \pi i / r}} \frac{v_{4}(t)}{t-z} d t+\int_{\rho e^{\pi i / r}}^{\rho e^{3 \pi i / r}} \frac{v_{2}(t)}{t-z} d t
$$

Substituting into (5.5) by means of this equation, we obtain

$$
\begin{aligned}
2 \pi i v_{0}(z)=C_{1} \int_{\rho e^{\pi i / r}}^{\infty e^{\pi i / r}} \frac{v_{1}(t)}{t-z} d t+C_{3} \int_{\rho e^{3 \pi i / r}}^{\infty e^{3 \pi i / r}} \frac{v_{3}(t)}{t-z} d t-\int_{\rho e^{-\pi i / r}}^{\rho e^{\pi i / r}} \frac{v_{0}(t)}{t-z} d t \\
-\int_{\rho e^{\pi i / r}}^{\rho e^{3 \pi i / r}} \frac{v_{2}(t)}{t-z} d t+\int_{\rho e^{-\pi i / r}}^{\infty e^{-\pi i / r}} \frac{v_{0}(t)}{t-z} d t-\int_{\rho e^{3 \pi i / r}}^{\infty e^{3 \pi i / r}} \frac{v_{4}(t)}{t-z} d t
\end{aligned}
$$

We may regard (5.5) as the first stage in a cycle of substitutions and (5.6) as the second stage. We may continue the substitutions until we reach the $r$ th stage, and then observe that

$$
\int_{\rho e^{-\pi i / r}}^{\infty e^{-\pi i / r}} \frac{v_{0}(t)}{t-z} d t=\int_{\rho e^{(2 r-1) \pi i / r}}^{\infty e^{(2 r-1) \pi i / r}} \frac{v_{2 r}(t)}{t-z} d t
$$

this being a consequence of the relation

$$
v_{2 r}(t)=v_{0}\left(t e^{-2 \pi i}\right)
$$

obtained from (4.1a) and (5.1). Therefore, (5.3) is precisely the result that is obtained on completion of the $r$ th stage. 
Lemma 5.2.

$2 \pi i a_{s, 1}=-\sum_{k=0}^{r-1} C_{2 k+1} \int_{\rho e^{(2 k+1) \pi i / r}}^{\infty e^{(2 k+1) \pi i / r}} v_{2 k+1}(t) t^{s} d t+\sum_{k=0}^{r-1} \int_{\rho e^{(2 k-1) \pi i / r}}^{\rho e^{(2 k+1) \pi i / r}} v_{2 k}(t) t^{s} d t$.

The derivation of this result from Lemma 5.1 is similar to the derivation of Lemma 3.2 of [12] from Lemma 3.1 of [12]. We omit the proof.

To complete the proof of Theorem 4.1, we observe from (1.16b) and (5.1) that for each $k$

$$
v_{2 k+1}(t)=e^{\xi_{2}(t)-\xi_{1}(t)} t^{\omega-1}\left\{\sum_{j=0}^{m-1} \frac{a_{j, 2}}{t^{j}}+\mathcal{O}\left(\frac{1}{t^{m}}\right)\right\}
$$

as $t \rightarrow \infty$ in $\hat{S}_{2 k+1}$ and, in particular, as $t \rightarrow \infty$ along the ray ph $t=(2 k+1) \pi / r$. By hypothesis, $\xi_{2}(t)-\xi_{1}(t)=t^{r}$, hence

$$
\begin{aligned}
\int_{\rho e^{(2 k+1) \pi i / r}}^{\infty e^{(2 k+1) \pi i / r}} v_{2 k+1}(t) t^{s} d t=\sum_{j=0}^{m-1} a_{j, 2} & \int_{\rho e^{(2 k+1) \pi i / r}}^{\infty e^{(2 k+1) \pi i / r}} e^{t^{r}} t^{s+\omega-j-1} d t \\
& +\int_{\rho e^{(2 k+1) \pi i / r}}^{\infty e^{(2 k+1) \pi i / r}} e^{t^{r}} \mathcal{O}\left(t^{s+\omega-m-1}\right) d t
\end{aligned}
$$

where the $\mathcal{O}$-term is uniform for all positive integer values of $s$. Talking a new variable $\tau$ defined by $t=\exp \{(2 k+1) \pi i / r\} \tau^{1 / r}$, we find that the right-hand side of the last equation becomes

$$
\begin{aligned}
\frac{e^{(2 k+1) \omega \pi i / r}}{r} \sum_{j=0}^{m-1} a_{j, 2} e^{(2 k+1)(s-j) \pi i / r} & \int_{\rho^{r}}^{\infty} e^{-\tau} \tau^{(s+\omega-j-r) / r} d \tau \\
& +\mathcal{O}\left(\int_{\rho^{r}}^{\infty} e^{-\tau} \tau^{(s+\Re \omega-n-r) / r} d \tau\right) .
\end{aligned}
$$

As in [12], $\S 4$, we may replace each lower limit $\rho^{r}$ by zero without disturbing the uniformity of the $\mathcal{O}$-term with respect to $s$. Thus, we obtain

$$
\begin{aligned}
\int_{\rho e^{(2 k+1) \pi i / r}}^{\infty e^{(2 k+1) \pi i / r}} v_{2 k+1}(t) t^{s} d t=\frac{e^{(2 k+1) \omega \pi i / r}}{r} & \sum_{j=0}^{m-1} a_{j, 2} e^{(2 k+1)(s-j) \pi i / r} \Gamma\left(\frac{s+\omega-j}{r}\right) \\
& +\mathcal{O}\left\{\Gamma\left(\frac{s+\Re \omega-m}{r}\right)\right\}, \quad s \rightarrow \infty . \quad
\end{aligned}
$$

To establish (4.18a), we substitute into the first sum in (5.7) by means of (2.3), (5.8) and then observe that the second sum is $\mathcal{O}\left(\rho^{s}\right)$ as $s \rightarrow \infty$ and therefore is absorbable in the estimate $\mathcal{O}[\Gamma\{(s+\Re \omega-m) / r\}]$. Lastly, in consequence of Stirling's formula, $\mathcal{O}[\Gamma\{(s+\Re \omega-m) / r\}] \subseteq \mathcal{O}[\Gamma\{(s+\omega-m) / r\}]$. Equation (4.18b) may be proved in a similar manner, and the proof of Theorem 4.1 is complete.

Remark. Using another approach, Immink [7] constructed integral representations of Stieltjes type for solutions of linear differential equations of any order; compare Lemma 5.1. From these representations, she then obtained asymptotic approximations for the Stokes multipliers that in effect supply the dominant terms of the expansions in Theorem 4.1. In other words, in the case of second-order equations, Immink's results correspond to ours with $m=1$. This is an important difference, however, because the restriction to $m=1$ renders the approximations much weaker for numerical purposes; compare the examples in $\S 6$ below. 


\section{Examples}

Example 1. We take

$$
\frac{d^{2} w}{d z^{2}}+\left(1-z^{2}\right) \frac{d w}{d z}+\left(1-z^{4}\right) w=0 .
$$

In the notation of $\S 1$, we have $f(z)=1-z^{2}, g(z)=1-z^{4}$, and hence $r=3$. For the formal series solutions (1.9), we find that

$$
\xi_{1}(z)=\frac{1-\sqrt{5}}{6} z^{3}+\left(\frac{\sqrt{5}}{10}-\frac{1}{2}\right) z, \quad \xi_{2}(z)=\frac{1+\sqrt{5}}{6} z^{3}-\left(\frac{\sqrt{5}}{10}+\frac{1}{2}\right) z
$$

and

$$
\mu_{1}=\frac{1}{5} \sqrt{5}-1, \quad \mu_{2}=-\frac{1}{5} \sqrt{5}-1, \quad \omega \equiv \mu_{2}-\mu_{1}=-\frac{2}{5} \sqrt{5} .
$$

Thus, the singulant $\xi_{2}(z)-\xi_{1}(z)=\frac{1}{3} \sqrt{5} z^{3}-\frac{1}{5} \sqrt{5} z$. Since this is not a monomial, Theorem 4.1 is not immediately applicable. Accordingly, we transform (6.1) as indicated in $\S 4$. On setting

$$
x^{3}=\frac{1}{3} \sqrt{5} z^{3}-\frac{1}{5} \sqrt{5} z
$$

and taking the cube root, we find that

$$
x=\frac{5^{\frac{1}{6}}}{3^{\frac{1}{3}}} z-\frac{1}{3^{\frac{1}{3}} 5^{\frac{5}{6}}} z^{-1}+\mathcal{O}\left(z^{-3}\right), \quad z \rightarrow \infty .
$$

Hence by reversion,

$$
z=\frac{3^{\frac{1}{3}}}{5^{\frac{1}{6}}} x+\frac{1}{3^{\frac{1}{3}} 5^{\frac{5}{6}}} x^{-1}+\mathcal{O}\left(x^{-3}\right), \quad x \rightarrow \infty,
$$

thus

$$
c_{0}=3^{\frac{1}{3}} 5^{-\frac{1}{6}}, c_{1}=0, c_{2}=3^{-\frac{1}{3}} 5^{-\frac{1}{6}}, c_{3}=0 .
$$

Our choice of new independent variable is therefore $\tilde{z}$, defined implicitly by

$$
z=\frac{3^{\frac{1}{3}}}{5^{\frac{1}{6}}} \tilde{z}+\frac{1}{3^{\frac{1}{3}} 5^{\frac{5}{6}}} \tilde{z}^{-1}
$$

compare (4.6). In the transformed differential equation

$$
\frac{d^{2} w}{d \tilde{z}^{2}}+\tilde{f}(\tilde{z}) \frac{d w}{d \tilde{z}}+\tilde{g}(\tilde{z}) w=0
$$

the coefficients are given by (4.9). We find that

Formal series solutions are

$$
\begin{aligned}
\tilde{f}(\tilde{z})=-c_{0}^{3} \tilde{z}^{2}+\frac{4}{5} c_{0}-\frac{4}{25 c_{0} \tilde{z}^{2}}+\frac{1}{125 c_{0}^{3} \tilde{z}^{4}}-\left(1-\frac{1}{5 c_{0}^{2} \tilde{z}^{2}}\right)^{-1} \frac{2}{5 c_{0}^{2} \tilde{z}^{3}}, \\
\tilde{g}(\tilde{z})=-c_{0}^{6} \tilde{z}^{4}-\frac{2}{5} c_{0}^{4} \tilde{z}^{2}+\frac{26}{25} c_{0}^{2}-\frac{46}{125 \tilde{z}^{2}}+\frac{26}{625 c_{0}^{2} \tilde{z}^{4}} \\
-\frac{2}{3125 c_{0}^{4} \tilde{z}^{6}}-\frac{1}{15625 c_{0}^{6} \tilde{z}^{8}} .
\end{aligned}
$$

$$
e^{\tilde{\xi}_{1}(\tilde{z})} \tilde{z}^{\mu_{1}} \sum_{s=0}^{\infty} \frac{\tilde{a}_{s, 1}}{\tilde{z}^{s}}, \quad e^{\tilde{\xi}_{2}(\tilde{z})} \tilde{z}^{\mu_{2}} \sum_{s=0}^{\infty} \frac{\tilde{a}_{s, 2}}{\tilde{z}^{s}},
$$

where $\mu_{1}$ and $\mu_{2}$ are as in (6.3) and

$$
\tilde{\xi}_{1}(\tilde{z})=-\frac{1}{2}\left(1-\frac{1}{\sqrt{5}}\right) \tilde{z}^{3}-\frac{2}{5} c_{0} \tilde{z}, \quad \tilde{\xi}_{2}(\tilde{z})=\frac{1}{2}\left(1+\frac{1}{\sqrt{5}}\right) \tilde{z}^{3}-\frac{2}{5} c_{0} \tilde{z} .
$$




\begin{tabular}{|l|c|c|c|c|}
\hline$s=33$ & $A_{0}$ & $A_{1}$ & $A_{2}$ & $A_{3}$ \\
\hline$m=1$ & $-4.28024360 i$ & $0.53504119+0.13182434 i$ & $-1.59057354-1.25459872 i$ & $-2.16118253 i$ \\
$m=10$ & $-4.87003491 i$ & $0.50940646+0.15648251 i$ & $-1.60891229-1.02017197 i$ & $-2.38919826 i$ \\
$m=19$ & $-4.87002156 i$ & $0.50940850+0.15647932 i$ & $-1.60891135-1.02017656 i$ & $-2.38916059 i$ \\
$m=28$ & $-4.87002160 i$ & $0.50940850+0.15647933 i$ & $-1.60891136-1.02017655 i$ & $-2.38916077 i$ \\
$m=37$ & $-4.87002160 i$ & $0.50940850+0.15647933 i$ & $-1.60891136-1.02017655 i$ & $-2.38916077 i$ \\
\hline
\end{tabular}

TABle 6.1. Approximations for the Stokes multipliers of (6.1).

In consequence, $\tilde{\xi}_{2}(\tilde{z})-\tilde{\xi}_{1}(\tilde{z})=\tilde{z}^{3}$ as expected and as required by (4.17).

With the aid of the software package MAPLE V [1], we computed numerically the Taylor series expansions of the functions $\tilde{p}_{j}(\tilde{z})$ and $\tilde{q}_{j}(\tilde{z})$ associated with (6.5); compare (1.13) and (1.14). From these results, we generated the coefficients $\tilde{a}_{s, 1}$ and $\tilde{a}_{s, 2}$ via (1.12), proceeding as far as $s=101$. Sample values are

$$
\begin{array}{rlrl}
\tilde{a}_{0,1} & =1, & \tilde{a}_{0,2} & =1, \\
\tilde{a}_{1,1} & =-0.39691860, & \tilde{a}_{1,2} & =0.25184962, \\
\tilde{a}_{2,1} & =0.033329236, & & \tilde{a}_{2,2}=-0.0872577074, \\
\tilde{a}_{3,1} & =-0.053431001, & \tilde{a}_{3,2} & =0.33387567, \\
\tilde{a}_{4,1} & =0.10293154, & \tilde{a}_{4,2} & =0.21695963, \\
\tilde{a}_{5,1} & =-0.029902385, & & \tilde{a}_{5,2}=-0.052190874 \\
\ldots & & \ldots \\
\tilde{a}_{101,1} & =-6.3158988 \times 10^{34}, & \tilde{a}_{101,2} & =1.2029605 \times 10^{35} .
\end{array}
$$

To compute the Stokes multipliers $\left\{\tilde{A}_{k}\right\}$ associated with (6.5), (4.19) and (4.21) were set up numerically with $s=33$ and various values of $m$ in (4.20) and (4.22). The two sets of three simultaneous linear algebraic equations were solved for the $\left\{\tilde{A}_{k}\right\}$. Subsequently, the desired multipliers $\left\{A_{k}\right\}$ for (6.1) were obtained by use of the identity

$$
A_{k}=\left(\frac{1}{3} \sqrt{5}\right)^{(-)^{k} \frac{2}{15} \sqrt{5}} \tilde{A}_{k}
$$

compare (4.16).

Final results for $m=1,10,19,28$, and 37 are presented in Table 6.1. Since $A_{4}=-\overline{A_{2}}$ and $A_{5}=-\overline{A_{1}}$ (compare (2.5) and (4.2)), we omit the results for $A_{4}$ and $A_{5}$. The agreement of the entries in the last two rows indicates that, to the present accuracy, the neglect of the $\mathcal{O}$-terms in equations (4.18) is justified after the stage $m=28$ is reached. We infer that these entries give the correct values of $A_{k}$ to eight decimal places.

Example 2. We take the first example in [10], which is the system

$$
x^{3} \frac{d W}{d x}=\left[\begin{array}{cc}
1+\frac{4}{3} x^{2} & \frac{1}{2} x \\
2 x & 2+x-x^{2}
\end{array}\right] W .
$$

In [10], the asymptotic analysis applies to the case $x \rightarrow 0$. In terms of $z=1 / x$, the system becomes

$$
\frac{d W}{d z}=\left[\begin{array}{cc}
-z-\frac{4}{3} z^{-1} & -\frac{1}{2} \\
-2 & -2 z-1+z^{-1}
\end{array}\right] W
$$


On decomposing $W=[X, Y]^{T}$, we obtain the simultaneous equations

$$
\frac{d X}{d z}+\left(z+\frac{4}{3 z}\right) X+\frac{1}{2} Y=0, \quad \frac{d Y}{d z}+2 X+\left(2 z+1-\frac{1}{z}\right) Y=0 .
$$

Eliminating $Y, X$, in turn, we find that

$$
\begin{aligned}
& \frac{d^{2} X}{d z^{2}}+\left(3 z+1+\frac{1}{3 z}\right) \frac{d X}{d z}+\left(2 z^{2}+z+\frac{5}{3}+\frac{4}{3 z}-\frac{8}{3 z^{2}}\right) X=0 \\
& \frac{d^{2} Y}{d z^{2}}+\left(3 z+1+\frac{1}{3 z}\right) \frac{d Y}{d z}+\left(2 z^{2}+z+\frac{8}{3}+\frac{4}{3 z}-\frac{1}{3 z^{2}}\right) Y=0
\end{aligned}
$$

Equations $(6.14 \mathrm{a}, \mathrm{b})$ each have an irregular singularity of rank 2 at infinity. On applying the theory of $\S 1$, we find that there are solutions $X_{k}, Y_{k}$ of $(6.14 \mathrm{a}, \mathrm{b})$, respectively, with the properties

$$
\begin{aligned}
k \text { even: } & X_{k} \sim e^{-z^{2}-z} z^{-1}, \quad Y_{k} \sim e^{-z^{2}-z}, \quad z \rightarrow \infty \text { in } \hat{S}_{k}, \\
k \text { odd: } & X_{k} \sim e^{-\frac{1}{2} z^{2}} z^{-\frac{1}{3}}, \quad Y_{k} \sim e^{-\frac{1}{2} z^{2}} z^{-\frac{4}{3}}, \quad z \rightarrow \infty \text { in } \hat{S}_{k},
\end{aligned}
$$

where $\hat{S}_{k}=\left\{z:\left(\frac{1}{2} k-\frac{3}{4}\right) \pi+\delta \leq \operatorname{ph} z \leq\left(\frac{1}{2} k+\frac{3}{4}\right) \pi-\delta\right\}$. (Thus $\sigma=0$ for both differential equations.) Moreover, from (6.15), it is clear the solution $Y$ of $(6.13 \mathrm{a}, \mathrm{b})$ that corresponds to $X=X_{k}$ is $Y=(-)^{k} 2 Y_{k}$, and also that

$$
\left[\begin{array}{cc}
X_{k} & X_{k-1} \\
(-)^{k} 2 Y_{k} & (-)^{k-1} 2 Y_{k-1}
\end{array}\right]
$$

is a fundamental matrix solution of (6.12). Any other fundamental matrix solution can be expressed in the form

$$
\left[\begin{array}{cc}
X_{k} & X_{k-1} \\
(-)^{k} 2 Y_{k} & (-)^{k-1} 2 Y_{k-1}
\end{array}\right] C
$$

where $C$ is a non-singular constant matrix $[17, \S 2.3]$. In particular,

$$
\left[\begin{array}{cc}
X_{k+1} & X_{k} \\
(-)^{k+1} 2 Y_{k+1} & (-)^{k} 2 Y_{k}
\end{array}\right]=\left[\begin{array}{cc}
X_{k} & X_{k-1} \\
(-)^{k} 2 Y_{k} & (-)^{k-1} 2 Y_{k-1}
\end{array}\right]\left[\begin{array}{ll}
C_{11}^{(k)} & C_{12}^{(k)} \\
C_{21}^{(k)} & C_{22}^{(k)}
\end{array}\right],
$$

say. We set ourselves the task of computing the scalar constants $C_{11}^{(k)}, C_{12}^{(k)}, C_{21}^{(k)}$, and $C_{22}^{(k)}$.

By comparing the asymptotic behavior of the two sides of (6.18) as $z \rightarrow \infty$ in the sector $\frac{1}{2} k-\frac{1}{4} \pi<\operatorname{ph} z<\frac{1}{2} k+\frac{1}{4} \pi$, we immediately conclude that $C_{11}^{(k)}=C_{k, X}=-C_{k, Y}$, $C_{12}^{(k)}=C_{21}^{(k)}=1$, and $C_{22}^{(k)}=0$. Here $\left\{C_{k, X}\right\}$ and $\left\{C_{k, Y}\right\}$ denote the "C-type" Stokes multipliers of equations $(6.14 \mathrm{a}, \mathrm{b})$, respectively; compare (2.2). Hence, (6.18) reduces to

$$
\left[\begin{array}{cc}
X_{k+1} & X_{k} \\
(-)^{k+1} 2 Y_{k+1} & (-)^{k} 2 Y_{k}
\end{array}\right]=\left[\begin{array}{cc}
X_{k} & X_{k-1} \\
(-)^{k} 2 Y_{k} & (-)^{k-1} 2 Y_{k-1}
\end{array}\right]\left[\begin{array}{cc}
C_{k, X} & 1 \\
1 & 0
\end{array}\right],
$$

and we also observe that the Stokes multipliers of $(6.14 a, b)$ differ only in sign.

We apply the method of $\S 4$ to compute the Stokes multipliers for (6.14a). We have $r=2$ and find that $\omega=\frac{2}{3}, c_{0}=\sqrt{2}, c_{1}=-1, c_{2}=\frac{1}{4} \sqrt{2}$. Accordingly, our transformation variable $\tilde{z}$ is defined by

$$
z=\sqrt{2} \tilde{z}-1
$$




\begin{tabular}{|l|c|c|c|c|}
\hline$s=30$ & $C_{0, X}$ & $C_{1, X}$ & $C_{2, X}$ & $C_{3, X}$ \\
\hline$m=1$ & $13.114172 i$ & $7.2724007-0.95787147 i$ & $-0.22234619-0.12837163 i$ & $4.4657414-5.8191481 i$ \\
$m=9$ & $14.849803 i$ & $7.2773761+0.30624016 i$ & $-0.20091135-0.11599622 i$ & $3.3734763-6.4555127 i$ \\
$m=17$ & $14.849851 i$ & $7.2773721+0.30622089 i$ & $-0.20091164-0.11599639 i$ & $3.3734910-6.4554995 i$ \\
$m=25$ & $14.849851 i$ & $7.2773721+0.30622084 i$ & $-0.20091164-0.11599639 i$ & $3.3734910-6.4554995 i$ \\
$m=33$ & $14.849851 i$ & $7.2773721+0.30622084 i$ & $-0.20091164-0.11599639 i$ & $3.3734910-6.4554995 i$ \\
\hline
\end{tabular}

TABLE 6.2. Approximations for the Stokes multipliers of (6.14a).

Since this is a linear transformation, the calculations are simpler than in Example 1. After computing the " $A$-type" Stokes multipliers $\left\{\tilde{A}_{k}\right\}$ of the transformed differential equation, the $C_{k, X}$ are obtained by use of the identity

$$
C_{k, X}=e^{(-)^{k}\left(\frac{1}{3} k \pi i-\frac{1}{2}\right)} 2^{(-)^{k} \frac{1}{3}} \tilde{A}_{k}
$$

compare (2.3) and (4.16).

Final results are presented in Table 6.2 for the case when $s=30$ in (4.19)-(4.22) and $m=1,9,17,25$, and 33 in (4.20) and (4.22). Similar conclusions may be drawn concerning accuracy to those drawn above for Table 6.1.

\section{Direct numerical methods for the Stokes multipliers}

In principle, the calculation of the Stokes multipliers $C_{k}$ and $A_{k}$ defined in $\S 2$ by direct integration of the differential equation (1.1) is quite simple. Let $z_{0}$ be a point in $S_{k-1}$ sufficiently close to infinity to enable $w_{k-1}\left(z_{0}\right)$ and $w_{k-1}^{\prime}\left(z_{0}\right)$ to be computed to a prescribed accuracy from (1.16a) or (1.16b) and their differentiated forms. Similarly, let $z_{1}$ be a point in the interior of $S_{k} \cup S_{k+1}$ that is sufficiently close to infinity to enable $w_{k}\left(z_{1}\right)$ and $w_{k+1}\left(z_{1}\right)$ to be computed from (1.16). By numerical integration, starting with the initial values $w_{k-1}\left(z_{0}\right)$ and $w_{k-1}^{\prime}\left(z_{0}\right)$, the value of $w_{k-1}\left(z_{1}\right)$ is obtained. Then

$$
C_{k}=\frac{w_{k+1}\left(z_{1}\right)-w_{k-1}\left(z_{1}\right)}{w_{k}\left(z_{1}\right)} \text {. }
$$

The difficulty in this approach is that it is prone to numerical instability. If $z_{1}$ is taken to be an interior point of $S_{k}$, then $w_{k-1}\left(z_{1}\right)$ and $w_{k+1}\left(z_{1}\right)$ will be almost indistinguishable numerically, and $w_{k}\left(z_{1}\right)$ will be exponentially small in comparison. This means that severe cancellation will occur in evaluating the right-hand side of (7.1), and consequently little or no precision will remain in the computed value of $C_{k}$. On the other hand, if $z_{1}$ is taken to be in $S_{k+1}$, then the computation of $w_{k-1}\left(z_{1}\right)$ will be unstable if the integration path lies in the neighborhood of infinity, because on parts of it (in fact within the left-hand half of $S_{k}$ ), $w_{k-1}(z)$ decays rapidly compared with other solutions of the differential equation.

The difficulty is overcome if the real part of the singulant $\xi_{2}(z)-\xi_{1}(z)$ is monotonic on the integration path. However, the construction of such a path is not straightforward in general. For reasons given above, it cannot be confined to the neighborhood of infinity, and it may be dependent on the distribution of turning points and the other singularities of the differential equation. In these cases, [14] may be helpful. Although this reference is devoted to the problem of calculating the Stokes multipliers asymptotically when there is a large parameter present in the differential equation, it includes an investigation of the topology of the curves along which $\Re\left\{\xi_{2}(z)-\xi_{1}(z)\right\}$ is monotonic. In addition, the Appendix in [14] supplies a numerical method for computing these curves. 
A modification to this procedure is to make the same preliminary transformation of independent variable as that described in $\S 4$ to reduce $\xi_{2}(z)-\xi_{1}(z)$ to the monomial form $z^{r}$. This greatly simplifies the task of finding integration paths on which $\Re\left\{\xi_{2}(z)-\xi_{1}(z)\right\}$ is monotonic, although it may do so at the expense of introducing an extra singularity into the differential equation at $z=0$.

Consider again Example 1 of $\S 6$. We may integrate numerically either (6.1) or (6.5). The only singularity of the former is at $z=\infty$, but the singulant is $\frac{1}{3} \sqrt{5} z^{3}-\frac{1}{5} \sqrt{5} z$. On the other hand, the latter equation has $\tilde{z}^{3}$ as singulant, but it has a second singularity at the origin. For illustration with this example, we compromise by integrating (6.1) numerically along rays through the origin on which $\Re\left(z^{3}\right)$ is monotonic. This combines the simplifying features of the two possibilities, but at the risk of introducing some instability into the computations.

In the numerical integration, we start with $z_{0}=11$ and we compute $w_{0}\left(z_{0}\right)$ and $w_{0}^{\prime}\left(z_{0}\right)$ by taking 11 terms in (1.16a) and its differentiated form. We obtain $w_{0}\left(z_{0}\right)=$ $1.0137883 \times 10^{-121}$ and $w_{0}^{\prime}\left(z_{0}\right)=-7.6141442 \times 10^{-120}$. By differentiation of (6.1), we see that the coefficients of the Taylor series expansion

$$
w_{0}(z)=\sum_{s=0}^{\infty} d_{s}\left(z-z_{0}\right)^{s}
$$

are given by $d_{0}=w_{0}\left(z_{0}\right), d_{1}=w_{0}^{\prime}\left(z_{0}\right)$, and

$$
\begin{aligned}
(s+1)(s+2) d_{s+2}=( & s+1)\left(z_{0}^{2}-1\right) d_{s+1}+\left(z_{0}^{4}+2 s z_{0}-1\right) d_{s} \\
& +\left(4 z_{0}^{3}+s-1\right) d_{s-1}+6 z_{0}^{2} d_{s-2}+4 z_{0} d_{s-3}+d_{s-4}
\end{aligned}
$$

$s=0,1,2, \ldots$, where we set $d_{s}=0$ if $s<0$. Taking the first 40 terms of (7.2) and its differentiated form, we approximate $w_{0}(z)$ and $w_{0}^{\prime}(z)$ at $z=z_{0}-\frac{1}{20}$. Again, we compute the Taylor series expansion of $w_{0}(z)$ at $z=z_{0}-\frac{1}{20}$, and with this expansion, we approximate $w_{0}(z)$ and $w_{0}^{\prime}(z)$ at $z=z_{0}-\frac{2}{20}$. After 220 steps, we obtain $w_{0}(0)=$ 0.81045660 and $w_{0}^{\prime}(0)=-0.16426746$. We then continue this process along the ray $\operatorname{ph} z=\frac{2}{3} \pi$. After 220 steps in this direction, we arrive at $z_{1}=11 \exp \left(\frac{2}{3} \pi i\right)$ with $w_{0}\left(z_{1}\right)=(3.8675043+3.3107321 i) \times 10^{311} .6$

To compute $C_{1}$ from (7.1), we also need $w_{1}\left(z_{1}\right)$ and $w_{2}\left(z_{1}\right)$. These quantities can be found by use of the expansions (1.16). Again with 11 terms, we obtain $w_{1}\left(z_{1}\right)=$ $(-8.2587430+4.8022021 i) \times 10^{311}$. In comparison, $w_{2}\left(z_{1}\right)$ is negligible. Accordingly, (7.1) yields

$$
C_{1}=0.17576723+0.50307920 i
$$

and, hence, from $(2.3)$, we obtain

$$
A_{1}=\exp \left(-\frac{2}{15} \sqrt{5} \pi i\right) C_{1}=0.50940850+0.15647933 i \text {. }
$$

This agrees with the result given in the last two rows in Table 6.1, indicating that the possible instability in integrating the differential equation along the chosen path was not realized.

Another general and reasonably stable variant of the method of numerical integration, and one that will extend more readily to differential equations of higher order, is as follows. As in Lemma 5.1, let $\rho$ again denote a constant that exceeds the radius of convergence of each of the power series (1.2a), (1.2b). Also, let $z_{j}(j=k-1, k, k+1)$

\footnotetext{
${ }^{6}$ This procedure for the numerical integration of $(6.1)$ is, of course, the well-known Taylor series method $[2, \S 6.3]$. We have used it simply for illustration; other methods could equally well be employed.
} 


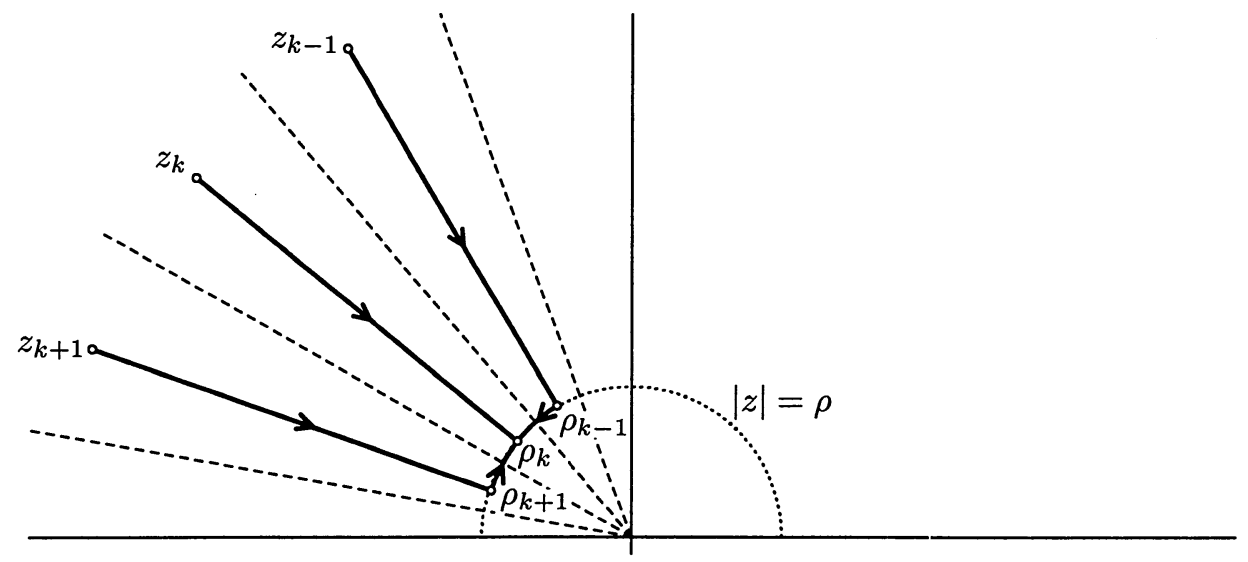

FiguRE 7.1. Paths for numerical integration (second method).

be a point in $S_{j}$ sufficiently close to infinity to enable $w_{j}\left(z_{j}\right)$ and $w_{j}^{\prime}\left(z_{j}\right)$ to be computed from (1.16a), (1.16b), their differentiated forms, and $\rho_{j}:=\rho \exp \left(i \mathrm{ph} z_{j}\right)$; see Figure 7.1. Starting with the initial values $w_{j}\left(z_{j}\right)$ and $w_{j}^{\prime}\left(z_{j}\right)$, vie integrate inwards along the ray $\operatorname{ph} z=\operatorname{ph} z_{j}$ to compute $w_{j}\left(\rho_{j}\right)$ and $w_{j}^{\prime}\left(\rho_{j}\right)$ for $j=k-1, k, k+1$. We then may compute $w_{k-1}\left(\rho_{k}\right)$ and $w_{k+1}\left(\rho_{k}\right)$ by integrating along arcs of the circle $|z|=\rho$. (This will be stable provided that $\rho$ can be chosen to be sufficiently small.) Lastly, $C_{k}$ is found from formula (7.1) with $z_{1}$ replaced by $\rho_{k}$ throughout.

All of the entries in Tables 6.1 and 6.2 were recomputed by at least one of the two methods described in the present section, and numerical agreement was satisfactory in all cases. We would add that the computations needed in these direct numerical methods are considerably longer than those used in the asymptotic method described in $\S 4$.

\section{Summary and conclusions}

In $\S 1$, we summarized the theory of the asymptotic solutions of the form

$$
e^{\xi_{1}(z)} z^{\mu_{1}} \sum_{s=0}^{\infty} \frac{a_{s, 1}}{z^{s}}, \quad e^{\xi_{2}(z)} z^{\mu_{2}} \sum_{s=0}^{\infty} \frac{a_{s, 2}}{z^{s}},
$$

in which $\xi_{1}(z)$ and $\xi_{2}(z)$ are polynomials, for linear second-order clifferential equations having an irregular singularity at $z=\infty$. In $\S 2$, we discussed the problem of finding the linear identities that hold among any three asymptotic solutions of these types. The coefficients in these identities are the so-called Stokes multipliers. The case of a singularity of rank unity was considered in $\S 3$, and asymptotic expansions of $a_{s, 1}$ and $a_{s, 2}$ for large $s$ were supplied in series of inverse factorials. These expansions then may be used to compute the Stokes multipliers quite simply.

In $\S 4$, the corresponding asymptotic expansions of $a_{s, 1}$ and $c_{s, 2}$ for large $s$ were stated for a singularity of arbitrary rank $r$, and it was shown how to use the results to compute the Stokes multipliers by solving two sets of $r$ simultaneous linear algebraic equations. $\S 5$, is devoted to the proof of the asymptotic expansions of the coefficients $a_{s, 1}$ and $a_{s, 2}$ stated in $\S 4$. Two numerical examples were furnished in $\S 6$, one for a singularity of rank 3 , the other for a system of two first-order differential equations having a singularity of rank 2 . 
In $\S 7$, we described another method for calculating the Stokes multipliers based on procedures for direct numerical integration of the differential equation. Potential problems of numerical instability were outlined, together with ways of overcoming this instability. The examples given in $\S 6$ were recomputed using this method.

Both methods we have described are very powerful, and both may be capable of extension to differential equations, or systems of differential equations, of order higher than two.

Acknowledgments. The authors are indebted to Professors D. A. Lutz and Y. Sibuya for supplying several references and Professor B. L. J. Braaksma for helpful discussions.

\section{References}

1. B. W. Char, K. O. Geddes, G. H. Gonnet, B. L. Leong, M. B. Monaghan, and S. M. Watt, Maple $V$ Library Reference Manual, Springer-Verlag, New York, 1991.

2. S. D. Conte and C. de Boor, Elementary Numerical Analysis: An Algorithmic Approach (3d ed.), McGraw-Hill, New York, 1980.

3. R. B. Dingle, Asymptotic Expansions: Their Derivation and Interpretation, Academic Press, London, 1973.

4. W. Gautschi, Norm estimates for inverses of Vandermonde matrices, Numer. Math. 23 (1975), 337-347.

5. F. L. Hinton, Stokes multipliers for a class of ordinary differential equations, J. Math. Phys. 20 (1979), 2036-2046.

6. R. Hoeppner, Über die Reihenreste der Lösungen linearen Differentialgleichungen in der Nähe stark singulärer Punkte, Ph. D. thesis, Universität Strasbourg, 1994.

7. G. K. Immink, A note on the relationship between Stokes multipliers and formal solutions of analytic differential equations, SIAM J. Math. Anal. 21 (1990), 782-792.

8. W. B. Jurkat, D. A. Lutz, and A. Peyerimhoff, Birkhoff invariants and effective calculations for meromorphic linear differential equations. I, J. Math. Anal. Appl. 53 (1976), 438-470.

9. __ Birkhoff invariants and effective calculations for meromorphic linear differential equations, II, Houston J. Math. 2 (1976), 207-238.

10. M. Loday-Richaud, Calcul des invariants de Birkhoff des systèmes d'ordre deux, Funkcial. Ekvac. 33 (1990), 161-225.

11. D. A. Lutz and R. Schäfke, Calculating connection coefficients for meromorphic differential equations, [Manuscript.]

12. A. B. Olde Daalhuis and F. W. J. Olver, Exponentially improved asymptotic solutions of ordinary differential equations. II Irregular singularities of rank one, Proc. Roy. Soc. London Ser. A 445 (1994), 39-56.

13. F. W. J. Olver, Asymptotics and Special Functions, Academic Press, New York, 1974.

14. - General connection formulae for Liouville-Green approximations in the complex plane, Philos. Trans. Roy. Soc. London Ser. A 289 (1978), 501-548.

15. F. W. J. Olver and F. Stenger, Error bounds for asymptotic solutions of second-order differential equations having an irregular singularity of arbitrary rank, SIAM J. Numer. Anal. Ser. B 2 (1965), 244-249.

16. W. J. Trjitzinsky, Analytic theory of linear differential equations, Acta Math. 62 (1933), 167-226.

17. W. Wasow, Asymptotic Expansions for Ordinary Differential Equations, Interscience, New York, 1965. Reprinted by Krieger Publishing Co., Huntington, New York, 1976.

18. C. Zhu and H. Nakamura, Stokes constants for a certain class of second-order ordinary differential equations, J. Math. Phys. 33 (1992), 2697-2717.

Institute for Physical Science and Technology, University of Maryland, College Park, MARYLAND 20742, U.S.A.

E-mail: aod@olgao.umd.edu, olver@bessel.umd.edu 